Working Papers

\title{
Institute of
}

Mathematical

\section{Economics}

\section{1}

January 2009

A representative individual from arrovian aggregation of parametric individual utilities

Frederik Herzberg

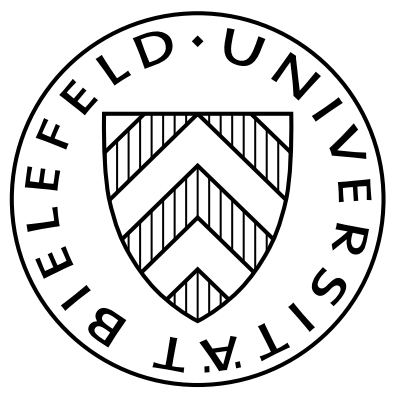




\title{
A REPRESENTATIVE INDIVIDUAL FROM ARROVIAN AGGREGATION OF PARAMETRIC INDIVIDUAL UTILITIES
}

\author{
FREDERIK HERZBERG
}

\begin{abstract}
This article investigates the representative-agent hypothesis for an infinite population which has to make a social choice from a given finite-dimensional space of alternatives. It is assumed that some class of admissible strictly concave utility functions is exogenously given and that each individual's preference ordering can be represented cardinally through some admissible utility function. In addition, we assume that (i) the class of admissible utility functions allows for a smooth parametrization, and (ii) the social welfare function satisfies Arrovian rationality axioms. We prove that there exists an admissible utility function $r$, called representative utility function, such that any alternative which maximizes $r$ also maximizes the social welfare function.
\end{abstract}

The proof utilizes a special nonstandard model of the reals, viz. the ultraproduct of the reals with respect to the ultrafilter of decisive coalitions; this construction explicitly determines the parameter vector of the representative utility function.

Key words: Representative individual; Arrovian social choice; ultrafilter; ultraproduct; nonstandard analysis.

Journal of Economic Literature Classification Number: D71.

2000 Mathematics Subject Classification Numbers: 91B14; $03 \mathrm{H} 05$.

Institut für Mathematische Wirtschaftsforschung, Universität Bielefeld, Universitätsstraße 25, D-33615 Bielefeld, Germany.

Department of Mathematics, University of California, Berkeley, CA 94720-3840, United States of America.

E-mail addresses: fherzberg@uni-bielefeld.de; herzberg@math.berkeley.edu. 


\section{INTRODUCTION}

The existence of a representative agent is as ubiquitious an assumption in macroeconomic theory as it is controversial, to say the least, from a microeconomic perspective (see Kirman 1992 and also Hartley 1996). Methodologically, the representative-agent hypothesis is of utmost importance for many branches of macroeconomics, since it provides a however tentative - microfoundation for models which otherwise would arguably be up in the air.

So far, there have been only few attempts at underpinning the representative-agent hypothesis itself. Clark (1992) has provided an indirect justification for certain representative utility functions through randomized social choice theory. A vindication for the representative consumer hypothesis - of course, without reference to normative social choice theory — can be found in a paper by Dow and Ribeiro da Costa Werlang (1988).

The usage of representative agent models in policy analysis can typically be of one of two kinds: Either one assumes the social planner's goal to be (1) the maximization of the social welfare function, or one postulates that the social planner aims at (2) the maximization of some other utility function which depends on the aggregate reaction of the population to his or her policy choices. The first situation is to some extent simpler and will be studied in the present paper.

Consider a population which has to make a social choice from a given finite-dimensional vector space of alternatives. Suppose that a set of strictly concave admissible utility functions is given, and that every individual's preference ordering can be represented by some admissible utility function. Assume that the population's collective decision procedure is given by a social welfare function in the sense of Arrovian social choice theory (Arrow 1963). We call an admissible utility function $r$ representative if and only if every alternative which maximizes $r$ also maximizes the social welfare function. If such a representative utility function exists, then the social 
planner can determine an optimal social choice simply by maximizing a single admissible utility function.

This paper constructs a representative individual utility function under the assumption that the set of alternatives is a finite-dimensional vector space. In addition, we assume that (i) the class of admissible utility functions can be smoothly parametrized and contains only strictly concave utility functions with well-posed maximization problems, and that (ii) the social welfare function satisfies Arrovian rationality axioms, which by results of Kirman and Sondermann (1972) ensure that the set of decisive coalitions is an ultrafilter.

The crucial step in our construction is the choice of a socially acceptable utility function: an admissible utility function whose maximum argument can never be overturned by any decisive coalition. The parameter of this socially acceptable utility function is explicitly derived from the sequence of parameters of the individual utility functions. (It is the image of that sequence under the canonical embedding into an ultraproduct of the parameter space with respect to the ultrafilter of decisive coalitions.) In fact, for any non-principal ultrafilter as the collection of decisive coalitions, one can establish the existence of a socially acceptable utility function under the above assumption (i), i.e. assuming the existence of a regular parametrization for the class of admissible utility functions (Theorem 1).

Utilizing assumption (ii) and the Kirman-Sondermann correspondence between Arrovian social welfare functions and ultrafilters (Kirman and Sondermann 1972), we prove that the socially acceptable utility function maximizes the social welfare function and is hence a representative utility function in the sense defined above (Theorem 2).

One can interpret this utility function as belonging to a representative agent, even though there does not need to be an actual individual in the population to which this utility function belongs. Therefore, our analysis clarifies the ontological status of the representative individual, thus 
responding to an objection raised by Kirman (1992) and Hartley (1996) against macroeconomic representative-agent models.

The existence proof for the socially acceptable (and hence representative) utility function is based on an interplay between the explicit use of ultrapowers and the implicit use of bounded ultrapowers through Robinsonian nonstandard analysis (Robinson 1966), both with respect to the non-principal ultrafilter of decisive coalitions. ${ }^{1}$

\section{MODEL FRAMEWORK}

In this paper, we consider the following model for a population which faces a social choice situation and whose individuals have cardinal preferences.

2.1. Population. We fix a set $N$, the population. Elements of $N$ are called individuals, subsets of $N$ coalitions. We fix some subset $\mathcal{D}$ of the power-set of $N$. The elements of $\mathcal{D}$ will be called decisive coalitions.

For our results, we shall later on assume that $\mathcal{D}$ is non-principal ultrafilter and hence that $N$ is infinite.

\subsection{Alternatives. We also fix a set $X$, the set of alternatives.}

For our results, we will have to assume that $X$ is a non-zero finitedimensional vector space; for simplicity, we shall assume $X=\mathbb{R}^{m}$ for some positive integer $m$.

2.3. Utilities. For every $i \in N$, we fix some function $u_{i}: X \rightarrow \mathbb{R}$, to be interpreted as the cardinal (individual) utility function of $i$. We define the utility profile as $\underline{u}:=\left\langle u_{i}\right\rangle_{i \in N}$.

We fix some class $\mathcal{M}$ of functions from $X$ to $\mathbb{R}$ such that $\left\{u_{i}\right\}_{i \in N} \subseteq \mathcal{M}$. The elements of $\mathcal{M}$ are called admissible utility functions.

\footnotetext{
${ }^{1}$ The connection between social choice theory and model theory, with a particular emphasis on ultrapowers, has been described Lauwers and Van Liedekerke (1995). Anderson (1991) is the standard introductory reference on nonstandard analysis in its applications to mathematical economics.
} 
For our results, we shall later on assume that $\mathcal{M}$ only consists of strictly concave utility functions (from $X=\mathbb{R}^{m}$ to $\mathbb{R}$ ) and that $\mathcal{M}$ can be smoothly parametrized.

\section{AxIOMS}

Having described the ontology of the model in Section 2, we now formulate assumptions which (i) ensure that our notion of decisive sets is consistent with the usual social choice terminology and (ii) allow us to construct a representative individual utility function.

Kirman and Sondermann (1972)[Theorem 1; Proposition 2] have shown that the collection of decisive coalitions generated by a social welfare function satisfying Arrovian rationality axioms is always a non-principal ultrafilter. We therefore require that $\mathcal{D}$ is a non-principal ultrafilter on $N$. This, however, is only possible if $N$ is infinite. Thus, our first axiom is:

Axiom 1 (Decisive Coalitions). $\mathcal{D}$ is a non-principal ultrafilter on $N$. In particular, $N$ is infinite.

The key assumption for the construction of a representative utility function is the existence of a sufficiently regular parametrization of the admissible utility functions. We now formulate the properties of this parametrization.

Axiom 2 (Parametrization). $X=\mathbb{R}^{m}$. Moreover, there exist $d \in \mathbb{N}$ and a twice continuously differentiable function $v: \mathbb{R}^{d+m} \rightarrow \mathbb{R}$ such that $\mathcal{M}=$ $\left\{v(\alpha ; \cdot): \alpha \in \mathbb{R}^{d}\right\}$. In particular, there is a sequence $\left\langle\alpha_{i}\right\rangle_{i \in N} \in\left(\mathbb{R}^{d}\right)^{N}$ such that $u_{i}=v\left(\alpha_{i} ; \cdot\right)$ for all $i \in N$.

We adopt the standard notation for derivatives: For each $x \in \mathbb{R}^{m}$, the first derivative of $v(\cdot ; x)$ is denoted by $\partial_{\alpha} v(\cdot ; x)$, and for each $\alpha \in \mathbb{R}^{d}$, the first and second derivatives of $v(\alpha ; \cdot)$ are denoted by $\partial_{x} v(\alpha ; \cdot)$ and $\partial_{x} \partial_{x} v(\alpha ; \cdot)$, respectively. 
For technical reasons, we need strict concavity of the representative utility function. To ensure this, we assume that all admissible utility functions are strictly concave. Also, our arguments that the representative utility function and all individual utility functions have a global maximum; therefore we assume that all admissible utility functions have global maxima. We denote the transpose of a vector $y$ by ${ }^{\top} y$.

Axiom 3 (Strict Concavity and Wellposedness). For all $u \in \mathcal{M}$ and $x, y \in$ $\mathbb{R}^{m}$ with $y \neq 0,{ }^{\top} y u^{\prime \prime}(x) y<0$. Moreover, every $u \in \mathcal{M}$ attains its global maximum on $\mathbb{R}^{m}$.

Of course, the Strict Concavity and Wellposedness conditions imply that $v(\alpha ; \cdot)$ even has a unique global maximum for every $\alpha \in \mathbb{R}^{d}$.

Finally, we need to ensure that the parameter of the representative utility function will be finite. This will be achieved by the following axiom:

Axiom 4 (Bounded Parameters). There exists some $R \in \mathbb{N}$ such that the coalition of all $i$ with $\left|\alpha_{i}\right|<R$ is decisive.

\section{A SOCIALLY ACCEPTABLE UTILITY FUNCTION}

An admissible utility function $r: \mathbb{R}^{m} \rightarrow \mathbb{R}$ is said to be $\mathcal{D}$-socially acceptable for $\underline{u}$ if and only if there exists some $\tilde{x} \in \mathbb{R}^{m}$ with $r(\tilde{x})=\max r$ such that for every $y \in \mathbb{R}^{m} \backslash\{\tilde{x}\}$, the coalition of $i$ with $u_{i}(\tilde{x})>u_{i}(y)$ is decisive.

The key to the aggregation of parametric individual utility functions into a representative utility function is the following result:

Theorem 1. Assuming Axioms 1,2,3,4, there exists some $\mathcal{D}$-socially acceptable utility function for $\underline{u}$.

\section{Aggregation}

We now link the model of Section 2 with classical Arrovian social choice theory. For this purpose, we first review some terminology from normative 
social choice theory, along the lines of the presentation in Kirman and Sondermann (1972).

5.1. Individual preferences. A relation $P \subseteq X \times X$ is called a weak order if and only if $P$ satisfies both of the following properties:

(1) $P$ is asymmetric: For all $x, y \in X$, if $x P y$ then not $y P x$.

(2) $P$ is negatively transitive: For all $x, y, z \in X$, if neither $x P y$ nor $y P z$ then also not $x P z$.

Herein, $x P y$ is shorthand for $\langle x, y\rangle \in P$, and should be read as ' $x$ is preferred to $y$ '. Let $\mathcal{P}$ be the set of weak orders on $X$. For all $x, y \in X$ and $\underline{P}=\left\langle P_{i}\right\rangle_{i \in N} \in \mathcal{P}^{N}$, we define

$$
C(x, y, \underline{P}):=\left\{i \in N: x P_{i} y\right\} .
$$

For $x \in X$ and $P \in \mathcal{P}, x$ will be called $P$-maximal if and only if $x P y$ for all $y \in X \backslash\{x\}$.

For $u: X \rightarrow \mathbb{R}$ and $P \in \mathcal{P}$, we say that $u$ is a cardinal utility representation of $P$ if and only if for all $x, y \in X$,

$$
u(x)>u(y) \Leftrightarrow x P y
$$

Given any $u$, there is obviously a unique $P$ whose cardinal utility representation is $u$. This relation will be denoted by $P^{u}$, and one easily verifies that $P^{u} \in \mathcal{P}$. Given an $N$-sequence $\underline{u}=\left\langle u_{i}\right\rangle_{i \in N}$ of functions from $X$ to $\mathbb{R}$ (as in Section 2), we define

$$
\underline{P}^{\underline{u}}:=\left\langle P^{u_{i}}\right\rangle_{i \in N} \in \mathcal{P}^{N}
$$

5.2. Social welfare functions. A social welfare function is a map $\sigma$ : $\mathcal{P}^{N} \rightarrow \mathcal{P}$. We say that a coalition $C \subseteq N$ is $\sigma$-decisive if and only if for all $x, y \in X$ and $\underline{P} \in \mathcal{P}^{N}$ one has $x \sigma(\underline{P}) y$ whenever $x P_{i} y$ for all $i \in C$ and $y P_{j} x$ for all $j \in N \backslash C$. The set of $\sigma$-decisive coalitions is denoted by $\mathcal{D}_{\sigma}$.

Consider now Arrow's rationality axioms for $\sigma$ : 
Axiom 5 (Unanimity Preservation; Pareto Principle). For all $x, y \in X$ and $\underline{P} \in \mathcal{P}^{N}$, if $C(x, y, \underline{P})=N$ then $x \sigma(\underline{P}) y$.

Axiom 6 (Independence of Irrelevant Alternatives). For all $x, y \in X$ and $\underline{P}, \underline{P}^{\prime} \in \mathcal{P}^{N}$, if both $C(x, y, \underline{P})=C\left(x, y, \underline{P}^{\prime}\right)$ and $C(y, x, \underline{P})=$ $C\left(y, x, \underline{P}^{\prime}\right)$ then

$$
x \sigma(\underline{P}) y \Leftrightarrow x \sigma\left(\underline{P}^{\prime}\right) y, \quad y \sigma(\underline{P}) x \Leftrightarrow y \sigma\left(\underline{P}^{\prime}\right) x .
$$

Axiom 7 (No Dictatorship). There exists no $i_{0} \in N$ such that for all $x, y \in$ $X$ and $\underline{P} \in \mathcal{P}^{N}$,

$$
x P_{i_{0}} y \Rightarrow x \sigma(\underline{P}) y \text {. }
$$

\section{SOCIAL WELFARE MAXIMIZATION AND}

\section{A REPRESENTATIVE UTILITY FUNCTION}

Kirman and Sondermann (1972) have established a correspondence between (i) Paretian, independent and non-dictatorial social welfare functions and (ii) non-principal ultrafilters. ${ }^{2}$ This correspondence allows us to translate Theorem 1 into the setting of normative social choice theory. Herein, an admissible utility function $r$ is called $\sigma$-representative of $\underline{P} \in$ $\mathcal{P}^{N}$ if and only if there exists some $\tilde{x} \in X$ with $r(\tilde{x})=\max r$ and any such $\tilde{x}$ is also $\sigma(\underline{P})$-maximal. (Whilst $r \in \mathcal{M}$ does not have a unique maximum in general, in our setting the uniqueness of $\tilde{x}$ follows from Axiom 3.)

Theorem 2. Let $\mathcal{D}=\mathcal{D}_{\sigma}$. Axiom 1 follows from Axioms 5,6,7 combined. Assuming Axioms 2,3,4,5,6,7, there exists an admissible utility function which is $\sigma$-representative of $\underline{P}^{\underline{u}}$.

\footnotetext{
${ }^{2}$ As elaborated by Kirman and Sondermann (1972), Hansson (1971, Postscript 1976), Armstrong (1980, 1985) and Schmitz (1977), even such non-dictatorial social welfare functions can still exhibit invisible dictatorship, viz. if and only if $N$ is endowed with a certain topological or measure-theoretic structure (e.g. a finite, countably additive measure).
} 
By the Parametrization axiom, the representative utility function of Theorem 2 can be written as $v(\tilde{\alpha} ; \cdot)$ for some $\tilde{\alpha} \in \mathbb{R}^{d}$. It can thus be interpreted as belonging to some "admissible" individual, be it an actual member of the population (in the case where $\tilde{\alpha}=\alpha_{i}$ for some $i \in N$ ) or just some imaginary individual which can be characterized by the parameter vector $\tilde{\alpha}$. For this reason, the representative utility function of Theorem 2 may also be called a representative individual utility function.

In the (trivial) special case where there exists some $\alpha_{0}$ such that $\left\{i \in N: \alpha_{i}=\alpha_{0}\right\}$ is decisive, $v\left(\alpha_{0} ; \cdot\right)$ is a representative utility function.

\section{CONCLUSION}

Given a parametric class of sufficiently regular admissible utility functions as well as a population with individual utilities from that class and some social structure on the population in terms of a family of decisive coalitions, we have establihed the existence of a socially acceptable utility function, i.e. an admissible utility function whose maximum argument will be supported against any other alternative by some decisive coalition.

If the social structure on the population is derived from a social welfare function satisfying Arrovian rationality axioms, then the socially acceptable utility function is even representative, i.e. its maximum argument also maximizes the social welfare function. It can be interpreted as being the utility function of some — actual or imaginary — "representative individual".

\section{APPENDiX A. PROOFS}

Proof of Theorem 1. Let $* \mathbb{R}:=\mathbb{R}^{N} / \mathcal{D}$ be the $\mathcal{D}$-ultrapower of $\mathbb{R}$, and let $*$ be the canonical embedding $z \mapsto\left[\langle z\rangle_{i \in N}\right]_{\mathcal{D}}$ from $\mathbb{R}$ into ${ }^{*} \mathbb{R}$. The map $*$ can be extended to a nonstandard embedding from the superstructure over $\mathbb{R}$ into the superstructure over $* \mathbb{R}$ (via a bounded ultrapower with respect to the non-principal ultrafilter $\mathcal{D}$, cf. Albeverio et al., 1986, Section 1.2). For the 
rest of this proof, we work in the resulting nonstandard universe. We have to construct some parameter $\tilde{\alpha}$ such that $v(\tilde{\alpha} ; \cdot)$ is $\mathcal{D}$-socially acceptable

Put $\bar{\alpha}:=\left[\left\langle\alpha_{i}\right\rangle_{i \in N}\right]_{\mathcal{D}} \in{ }^{*} \mathbb{R}^{d}$, and let $\tilde{\alpha}:={ }^{\circ} \bar{\alpha}$. This standard part exists in $\mathbb{R}^{d}$ due to the Boundedness axiom. Applying the transfer principle of nonstandard analysis to the Wellposedness axiom, we learn that ${ }^{*} v(\bar{\alpha} ; \cdot)$ attains its global maximum in some $\bar{x} \in{ }^{*} \mathbb{R}^{m}$. However, applying the transfer principle to the first-order condition

$\forall \alpha \in \mathbb{R}^{d} \quad \forall x \in \mathbb{R}^{m} \quad\left(\left(\forall y \in \mathbb{R}^{m} \quad v(\alpha ; y) \leq v(\alpha ; x)\right) \Rightarrow \partial_{x} v(\alpha ; x)=0\right)$ we find that ${ }^{*} \partial_{x} v(\bar{\alpha} ; \bar{x})=0$.

Consider now the map $w$ which assigns to each $\alpha \in \mathbb{R}^{d}$ the unique $x=$ $w(\alpha) \in \mathbb{R}^{m}$ such that $\partial_{x} v(\alpha ; x)=0$. (Existence and uniqueness follow from the Strict Concavity and Wellposedness axiom.) On the one hand, the transfer principle yields that

$$
\forall \alpha \in{ }^{*} \mathbb{R}^{d} \quad \forall x \in{ }^{*} \mathbb{R}^{m}\left(x={ }^{*} w(\alpha) \Leftrightarrow{ }^{*} \partial_{x} v(\alpha ; x)=0\right)
$$

hence $\bar{x}={ }^{*} w(\bar{\alpha})$. On the other hand, the implicit function theorem teaches that $w$ is continuously differentiable and thus continuous, whence ${ }^{*} w$ is $S$ continuous. Since $\bar{\alpha}$ is finite, it follows that $\bar{x}={ }^{*} w(\bar{\alpha})$ is finite, too. We put $\tilde{x}:={ }^{\circ} \bar{x}$.

Due to the Parametrization axiom, $v$ is continuous and hence ${ }^{*} v$ is $S$ continuous. Therefore, we have for all $y \in \mathbb{R}^{m}$,

(1) $v(\tilde{\alpha} ; y)-v(\tilde{\alpha} ; \tilde{x}) \simeq{ }^{*} v(\bar{\alpha} ; y)-{ }^{*} v(\bar{\alpha} ; \tilde{x}) \simeq{ }^{*} v(\bar{\alpha} ; y)-{ }^{*} v(\bar{\alpha} ; \bar{x})$.

In the next step, we combine Taylor's theorem with the Strict Concavity axiom, to see that the right-hand side of Equation (1) has a strictly negative standard part: Applying the transfer principle to Taylor's theorem yields a finite $\xi \in{ }^{*} \mathbb{R}^{m}$ such that

$$
\begin{aligned}
& { }^{*} v(\bar{\alpha} ; y)-{ }^{*} v(\bar{\alpha} ; \bar{x}) \\
& =^{\top}(y-\bar{x})^{*} \partial_{x} v(\bar{\alpha} ; \bar{x})+\frac{1}{2}{ }^{\top}(y-\bar{x})^{*} \partial_{x} \partial_{x} v(\bar{\alpha} ; \xi)(y-\bar{x}),
\end{aligned}
$$


hence

$$
{ }^{*} v(\bar{\alpha} ; y)-{ }^{*} v(\bar{\alpha} ; \bar{x})=\frac{1}{2}{ }^{\top}(y-\bar{x})^{*} \partial_{x} \partial_{x} v(\bar{\alpha} ; \xi)(y-\bar{x})
$$

(since $\bar{x}$ is a local maximum of ${ }^{*} v(\bar{\alpha} ; \cdot)$ ). Now, $\partial_{x} \partial_{x} v$ is continuous by the Parametrization axiom, so ${ }^{*} \partial_{x} \partial_{x} v$ is $S$-continuous and therefore ${ }^{*} \partial_{x} \partial_{x} v(\bar{\alpha} ; \xi) \simeq \partial_{x} \partial_{x} v\left(\tilde{\alpha} ;{ }^{\circ} \xi\right)$, which is negative definite by the Strict Concavity axiom. It follows that

$$
\begin{aligned}
{ }^{*} v(\bar{\alpha} ; y)-{ }^{*} v(\bar{\alpha} ; \bar{x}) & \simeq \frac{1}{2}^{\top}(y-\bar{x}) \partial_{x} \partial_{x} v\left(\tilde{\alpha} ;{ }^{\circ} \xi\right)(y-\bar{x}) \\
& \simeq \frac{1}{2}{ }^{\top}(y-\tilde{x}) \partial_{x} \partial_{x} v\left(\tilde{\alpha} ;{ }^{\circ} \xi\right)(y-\tilde{x})
\end{aligned}
$$

and thus

$$
\forall y \in \mathbb{R}^{m} \backslash\{\tilde{x}\} \quad{ }^{\circ}\left({ }^{*} v(\bar{\alpha} ; y)-{ }^{*} v(\bar{\alpha} ; \bar{x})\right)<0 .
$$

Comparing this result with Equation (1), we lastly obtain that $v(\tilde{\alpha} ; y)-$ $v(\tilde{\alpha} ; \tilde{x})<0$ for all standard $y \neq \tilde{x}$. This proves that $\tilde{x}$ is the unique maximum of $v(\tilde{\alpha} ; \cdot)$.

In order to verify that $v(\tilde{\alpha} ; \cdot)$ is $\mathcal{D}$-socially acceptable, we still need to show that for any given $y \in \mathbb{R}^{m} \backslash\{\tilde{x}\}$, the set of all $i \in N$ with $u_{i}(\tilde{x})>$ $u_{i}(y)$ is decisive (i.e. $\left.\in \mathcal{D}\right)$. Define a function $t$ by $t(z):=v(z ; \tilde{x})-v(z ; y)$ for all $z \in \mathbb{R}^{d}$, whence

$$
\begin{aligned}
\left\{i \in N: u_{i}(\tilde{x})>u_{i}(y)\right\} & =\left\{i \in N: v\left(\alpha_{i} ; \tilde{x}\right)-v\left(\alpha_{i} ; y\right)>0\right\} \\
& =\left\{i \in N: t\left(\alpha_{i}\right)>0\right\} .
\end{aligned}
$$

Due to the construction of the nonstandard embedding $*$ via the bounded ultrapower (with respect to $\mathcal{D}$ ) of the superstructure over the reals, one has the equivalence $\left\{i \in N: t\left(\alpha_{i}\right)>0\right\} \in \mathcal{D} \Leftrightarrow{ }^{*} t(\bar{\alpha})>0$ which through Equation (3) yields

$$
\left\{i \in N: u_{i}(\tilde{x})>u_{i}(y)\right\} \in \mathcal{D} \Leftrightarrow{ }^{*} t(\bar{\alpha})>0 .
$$

However, by applying the transfer principle to the defining equation for $t$, we get ${ }^{*} t(z)={ }^{*} v(z ; \tilde{x})-{ }^{*} v(z ; y)$ for all $z \in{ }^{*} \mathbb{R}^{d}$, so ${ }^{*} t(\bar{\alpha})={ }^{*} v(\bar{\alpha} ; \tilde{x})-$ 
${ }^{*} v(\bar{\alpha} ; y)$ and therefore (by the $S$-continuity of ${ }^{*} v$ ),

$$
{ }^{*} t(\bar{\alpha})={ }^{*} v(\bar{\alpha} ; \tilde{x})-{ }^{*} v(\bar{\alpha} ; y) \simeq{ }^{*} v(\bar{\alpha} ; \bar{x})-{ }^{*} v(\bar{\alpha} ; y)
$$

The standard part of the right-hand side is strictly positive by inequality (2), and therefore ${ }^{\circ}\left({ }^{*} t(\bar{\alpha})\right)>0$, too. Hence, ${ }^{*} t(\bar{\alpha})>0$, and by equivalence (4) finally

$$
\left\{i \in N: u_{i}(\tilde{x})>u_{i}(y)\right\} \in \mathcal{D} \text {. }
$$

Proof of Theorem 2. Kirman and Sondermann (1972)[Theorem 1; Proposition 2] have shown that $\mathcal{D}_{\sigma}$ is a non-principal ultrafilter whenever $\sigma$ satisfies Axioms 5,6,7. Hence, Axiom 1 follows from Axioms 5,6,7 for $\mathcal{D}=\mathcal{D}_{\sigma}$.

Now suppose Axioms 2,3,4,5,6,7 and hence also Axiom 1 are satisfied (for $\mathcal{D}=\mathcal{D}_{\sigma}$ ). Then, Theorem 1 ensures the existence of $\tilde{\alpha} \in$ $\mathbb{R}^{d}$ and $\tilde{x} \in \mathbb{R}^{m}=X$ such that $v(\tilde{\alpha} ; \tilde{x})=\max v(\tilde{\alpha} ; \cdot)$ and $\left\{i \in N: u_{i}(\tilde{x})>u_{i}(y)\right\} \in \mathcal{D}_{\sigma}$ for every $y \in X \backslash\{\tilde{x}\}$. Fix an arbitrary $y \in X \backslash\{\tilde{x}\}$. For all $i \in N$ with $u_{i}(\tilde{x})>u_{i}(y)$ we have $\tilde{x} P^{u_{i}} y$ and thus $\tilde{x} P_{i}^{\underline{u}} y$ by definition. Therefore,

$$
\left\{i \in N: \tilde{x} P_{i}^{\underline{u}} y\right\} \supseteq\left\{i \in N: u_{i}(\tilde{x})>u_{i}(y)\right\} \in \mathcal{D}_{\sigma} .
$$

Since $\mathcal{D}_{\sigma}$ is an ultrafilter and hence closed under supersets, also

$$
\left\{i \in N: \tilde{x} P_{i}^{\underline{u}} y\right\} \in \mathcal{D}_{\sigma} .
$$

Kirman and Sondermann (1972)[Theorem 1(i)] have shown that this already implies $\tilde{x} \sigma\left(\underline{P}^{\underline{u}}\right) y$. Since $y$ was an arbitrary element of $X \backslash\{\tilde{x}\}$, we obtain that $\tilde{x} \sigma\left(\underline{P}^{\underline{u}}\right) y$ for every $y \in X \backslash\{\tilde{x}\}$.

\section{ApPEndiX B. COMments On METHODOLOGY}

Ultrafilters have been applied extensively in social choice theory for nearly four decades: First, Fishburn (1970) proved that the axioms in 
Arrow's social choice theorem ${ }^{3}$ are consistent for economies with infinitely many individuals, although they are inconsistent for economies with finitely many individuals. Hansson (1971, Postscript 1976) strenghened these results. In the seminal work by Kirman and Sondermann (1972), it was shown that under the assumption of some measure-theoretic structure on the set of individuals, Arrow's axioms can lead, even for economies with infinitely many individuals, to an invisible dictator, i.e. to a decreasing sequence of winning coalitions with arbitrarily small measure. Armstrong (1980, 1985) generalized Kirman and Sondermann's (1972) results to measure spaces of individuals. Other generalizations of Kirman and Sondermann's (1972) analysis were discovered by Grafe and Grafe (1983) as well as (for intergenerational economies) Campbell (1990). Schmitz (1977) showed that Kirman and Sondermann's (1972) results cannot be extended to spaces of individuals that are endowed with an infinite measure, and Hansson (1976) pointed out that for some topologies on the population set, there will even be multiple invisible dictators, which seems to stretch the term "dictator" too far.

Ultraproducts - a model-theoretic construction based on ultrafilters (cf. e.g. Chang and Keisler, 1973) - entered social choice theory when Lauwers and Van Liedekerke (1995) used them to prove that there is a oneto-one correspondence between non-dictatorial Arrow-type aggregation functions and non-principal ultrafilters. ${ }^{4}$

\footnotetext{
${ }^{3}$ These axioms are: the existence of at least three alternatives, transitivity (i.e. unrestricted domain), independence, the Pareto principle, and non-dictatorship.

${ }^{4}$ In spite of this correspondence between Arrow aggregation functions and ultrafilters, Lauwers and Van Liedekerke (1995) argued in the conclusion of their paper that aggregation should not be formalized via ultrafilters in the context of social choice (pp. 235-236): First, they claim that ultrafilters exhibit “insuperable arbitrariness" (p. 236). As an example, they point to the well-known fact that every ultrafilter $\mathcal{D}$ on the set of natural numbers must, because of its maximality, either claim that "DD-almost all" positive integers are even or claim that "DD-almost all" integers are odd. (Herein, a property is set to hold for $\mathcal{D}$-almost all $i$ if and only if the set of all $i$ for which that property holds is in
} 
Some other applications of ultrafilters and ultraproducts in the social sciences, with a particular emphasis on de Condorcet's (1785) paradox, were recently reviewed by Haddad (2005). The existence of non-principal ultrafilters, under the assumption of the Axiom of Choice, was established by Ulam (1929) and Tarski (1930); the main results on ultraproducts are due to Łoś (1955).

In this paper, the ultraproduct methodology is used to build a socialchoice theoretic foundation for representative-agent models for populations with parametric individual utilities. We consider an infinite population $N$ of individuals whose utilities are functions of the social planner's policy. We suppose that these functions can be parametrized in a smooth and strictly concave manner. We consider an exogeneously given system of decisive coalitions which form a non-principal ultrafilter $\mathcal{D}$ on $N$; note that every non-dictatorial Arrovian social welfare function on an infinite population induces such a $\mathcal{D}$ (Fishburn 1970, Hansson 1976). The role of the social planner is to implement the social choice of the population. In other words, the social planner must maximize, simultaneously, the utility of each individual in some decisive coalition. We show that this problem

D.) This also illustrates their second objection, viz. that ultrafilters do not represent our intuition of "almost all" (p. 236).

From a foundational stance, the first point seems well-taken at first sight, since the ultrafilter existence theorem is a consequence of the Axiom of Choice. However, the ultrafilter existence theorem is not equivalent to the Axiom of Choice, as shown by Halpern and Levy (1971) (cf. also Banaschewski 1983), and in special social choice problems, ultrafilters may even be viewed as given exogeneously (modelling collections of coalitions). The fact that ultrafilters do not necessarily formalize our notion of "almost all" is no ultimate challenge for their use in social choice theory, since collective decision making sometimes ignores significant minorities.

In this paper, in particular, we use an ultrafilter on the population set to model the set of those coalitions which determine the social planner's policy. Hence, the ultrafilter is an exogeneous variable of the model, and it only captures a subjective notion of "most" (from the social planner's vantage point), not a concept of "almost all". 
can be reduced to maximizing just one element of the parametrized set of individual utility functions. One can interpret this utility function as belonging to a representative agent, even though there does not need to be an actual individual in the population to which this utility function belongs. In this sense, our results clarify the ontological status of the representative agent.

The proof of Theorem 1 employs both a classical ultrapower construction and implicitly also a bounded ultrapower of a superstructure (through Robinsonian nonstandard analysis) — both with respect to the non-principal ultrafilter of all decisive coalitions among the population. The description of our model and the statement of our results, however, do not assume any knowledge of model theory or nonstandard analysis.

For an economically motivated introduction into model theory and ultrapowers, we refer to Lauwers and Van Liedekerke (1995). Anderson (1991) provides an introduction to nonstandard analysis with an emphasis on applications in mathematical economics.

\section{REFERENCES}

Albeverio, S., Fenstad, J., Høegh-Krohn, R., and Lindstrøm, T. (1986). Nonstandard methods in stochastic analysis and mathematical physics. Pure and Applied Mathematics. 122. Orlando, FL: Academic Press. Anderson, R. (1991). Non-standard analysis with applications to economics. In Hildenbrand, W. and Sonnenschein, H., editors, Handbook of mathematical economics. Volume IV, pages 2145-2208. Handbooks in Economics. Amsterdam: North-Holland.

Armstrong, T. (1980). Arrow's theorem with restricted coalition algebras. Journal of Mathematical Economics, 7(1):55-75.

Armstrong, T. (1985). Precisely dictatorial social welfare functions. Erratum and addendum to: "Arrow's theorem with restricted coalition algebras". Journal of Mathematical Economics, 14(1):57-59. 
Arrow, K. (1963). Social choice and individual values. 2nd ed. Cowles Commission Monograph. 12. New York, NY: Wiley.

Banaschewski, B. (1983). The power of the ultrafilter theorem. Journal of the London Mathematical Society. Second Series, 27(2):193-202.

Campbell, D. (1990). Intergenerational social choice without the Pareto principle. Journal of Economic Theory, 50(2):414-423.

Chang, C. and Keisler, H. (1973). Model theory. Studies in Logic and the Foundations of Mathematics. 73. Amsterdam: North-Holland.

Clark, S. (1992). The representative agent model of probabilistic social choice. Mathematical Social Sciences, 23(1):45-66.

Dow, J. and Ribeiro da Costa Werlang, S. (1988). The consistency of welfare judgements with a representative consumer. Journal of Economic Theory, 44(2):269-280.

Fishburn, P. (1970). Arrow's impossibility theorem: concise proof and infinite voters. Journal of Economic Theory, 2(1):103-106.

Grafe, F. and Grafe, J. (1983). On Arrow-type impossibility theorems with infinite individuals and infinite alternatives. Economics Letters, 11(12):75-79.

Haddad, L. (2005). Un outil incomparable: l'ultrafiltre. Tatra Mountains Mathematical Publications, 31:131-176.

Halpern, J. and Levy, A. (1971). The Boolean prime ideal theorem does not imply the axiom of choice. In Axiomatic Set Theory. Proceedings of Symposia in Pure Mathematics. XIII. Part 1, pages 83134. Providence, RI: American Mathematical Society.

Hansson, B. (1976). The existence of group preference functions. Public Choice, 38:89-98.

Hartley, J. (1996). Retrospectives: The origins of the representative agent. Journal of Economic Perspectives, 10(2):169-177.

Kirman, A. (1992). Whom or what does the representative individual represent? Journal of Economic Perspectives, 6(2):117-136. 
Kirman, A. and Sondermann, D. (1972). Arrow's theorem, many agents, and invisible dictators. Journal of Economic Theory, 5(2):267-277.

Lauwers, L. and Van Liedekerke, L. (1995). Ultraproducts and aggregation. Journal of Mathematical Economics, 24(3):217-237.

Łoś, J. (1955). Quelques remarques, théorèmes et problèmes sur les classes définissables d'algèbres. In Skolem, T., Hasenjaeger, G., Kreisel, G., Robinson, A., Wang, H., Henkin, L., and Łoś, J., editors, Mathematical Interpretation of Formal Systems, pages 98-113. Studies in Logic and the Foundation of Mathematics. Amsterdam: North- Holland.

Marquis de Condorcet, M. (1785). Essai sur l'application de l'analyse à la probabilité des décisions à la pluralité. Imprimerie Royale.

Robinson, A. (1966). Non-standard analysis. Studies in Logic and the Foundations of Mathematics. Amsterdam: North-Holland.

Schmitz, N. (1977). A further note on Arrow's impossibility theorem. Journal of Mathematical Economics, 4(2):189-196.

Tarski, A. (1930). Une contribution à la théorie de la mesure. Fundamenta Mathematicae, 15:42-50.

Ulam, S. (1929). Concerning functions of sets. Fundamenta Mathematicae, 14:231-233. 